\title{
Evaluation of serum adenosine deaminase activity and autoantibodies in systemic lupus erythematosus
}

\author{
Corresponding author: \\ Seraj Ahmed Khan \\ Department of Biochemistry, \\ BP Koirala Institute of Health Sciences, \\ Dharan, Nepal \\ e-mail: drserajkhan@gmail.com
}

Medical Research Journal 2020;

Volume 5, Number 3, 154-157 10.5603/MRJ.a2020.0031

Copyright (C) 2020 Via Medica ISSN 2451-2591

\begin{abstract}
Introduction: Systemic lupus erythematosus (SLE) is a complex autoimmune disease with no single specific and sensitive test available for its diagnosis. Adenosine deaminase (ADA) is an enzyme that can act as an indicator of cellular immunity reflecting the extent of inflammation. The aim of this study was to investigate the role of serum adenosine deaminase activity and its association with antinuclear antibodies (ANA) and anti-dsDNA (anti-double stranded deoxyribonucleic acid) in SLE patients.

Material and methods: In this study, we included 36 diagnosed case of SLE as per the American Rheumatology Association (ARA) criteria and 30 healthy control. Serum ADA activity was measured by a spectrophotometric technique based on Giusti and Gallanti. ANA and Anti ds-DNA were measured by indirect Enzyme-Linked Immune Sorbent Assay (ELISA). Normal values of serum ADA activity, ANA and anti ds-DNA was $<25 \mathrm{IU} / \mathrm{L},<10 \mathrm{U} / \mathrm{mL}$ and $<25 \mathrm{IU} / \mathrm{mL}$ respectively.

Results: The mean ADA activity $(37.03 \pm 13.03 \mathrm{IU} / \mathrm{L})$ in SLE patients was higher as compared to control $(18.23 \pm 10.12 \mathrm{IU} / \mathrm{L})$, and the difference was statistically significant $(p<0.001)$. Median values of ANA and anti-dsDNA in SLE patients was $16.9(8.5,42.8)$ and $24.7(20.5,45.0)$ respectively. Serum ANA was positive in 25 out of 36 cases, anti-dsDNA was positive in 17 out of 36 cases and ADA was positive in 29 out of 36 cases. We observed a weak positive correlation between ANA and anti-dsDNA $(r=0.46$, $p=0.005)$, whereas a moderate correlation between ANA and ADA ( $r=0.525, p=0.001)$ in SLE patients. Conclusion: Serum ADA was significantly higher in SLE patients as compared to control and more specific than autoantibodies. Determination of ADA activity is a reliable, cost-effective, and easy test that can be used as an alternative parameter for diagnosing and evaluating disease activity in SLE patients. Key words: SLE, ADA, ANA, anti-dsDNA
\end{abstract}

Med Res J 2020; 5 (3): 154-157

\section{Introduction}

Adenosine deaminase is a hydrolytic enzyme that catalyzes the conversion of adenosine to inosine or 2'-deoxyadenosine to 2'-deoxyadenosine and is widely distributed in many tissues [1]. The role of ADA has been widely discussed and its role in regulating immune response has been demonstrated experimentally. Numerous evidence highlights its role in the function, maturation and regulation of immunological responses [2-5]. Impaired immune tolerance is an important characteristic of autoimmune diseases, which led many researchers to investigate the role of ADA in autoimmune disease [6, 7]. Several studies have shown increased ADA activity in diseases like rheumatoid arthritis (RA), and SLE [8-11]. Autoimmune disease can be organ-specific or non-organ-specific, in which the body damages self-tissues [12]. SLE is a connective tissue disease of idiopathic origin, the spectrum of which covers a wide array of clinical and laboratory manifestations. While the etiology of SLE is thought to be multifactorial, the disease is characterized by the production of autoantibodies. SLE is a prototypic example of non-organ specific autoimmune disorder in which plethora of autoantibodies is formed especially against nuclear DNA. Moreover, T-cell/B-cell dysregulation are an important hallmark of SLE $[13,14]$. Though the 5-year survival rate is $90 \%$ [15], however, survival rate has not improved since 1980. Due to its multi-organ involvement of SLE no single specific test is available till date in making its diagnosis. Hence it becomes crucial to look for a test that can be more specific and sensitive 
in diagnosis and monitoring disease activity. Therefore this study was undertaken to assess the role of serum ADA level in SLE patients and explore its role in the diagnosis of the same.

\section{Material and methods}

\section{Study population and setting}

This was a hospital-based cross-sectional study conducted in the Department of Biochemistry in collaboration with the Department of Dermatology and Venerology. A total of 36 diagnosed cases of SLE patients and 30 age and sex-matched healthy control were included in the study. A brief interview was taken from the patient and control group to fill up the preformed proforma.

\section{Ethical clearance}

The research proposal was approved by the Institutional Review Committee, of B.P. Koirala Institute of Health Sciences, Dharan, Nepal and was conducted in accordance with the Declaration of Helsinki adopted by the $18^{\text {th }}$ World Medical Assembly, Helsinki, Finland, June 1964. The study was initiated only after taking the written informed consent from the participants.

\section{Sample collection and analysis}

Three $\mathrm{mL}$ venous blood was drawn in a plain vial following the standard protocol. The samples were collected in the Immunoassay Laboratory and the serum was separated after centrifuging the sample for 10 minutes at $3000 \mathrm{rpm}$. Collected serum was used for the analysis of ADA, ANA, anti-dsDNA and other biochemical parameters

\section{Analytical procedures}

Serum ADA activity: The ADA activity was measured at $37^{\circ} \mathrm{C}$ according to the method of Giusti and Galanti, based on the Bertholet reaction, i.e. the formation of colored indophenol complexes from ammonia liberated from adenosine, and quantified spectrophotometrically at $625 \mathrm{~nm}$. ANA and anti-dsDNA were measured by indirect Enzyme-Linked Immunosorbent Assay (ELISA). As per our Lab, normal values of serum ADA activity, ANA and anti-dsDNA were $<25 \mathrm{IU} / \mathrm{L},<10 \mathrm{U} / \mathrm{mL}$ and $<25 \mathrm{IU} / \mathrm{mL}$ respectively.

\section{Statistical analysis}

The values are expressed as mean $\pm S D$, median and interquartile range for continuous variable and number and percentage for nominal variables. Normality of the data was tested by the Kolmogorov Smirnov test. Statistical analysis of differences within and between the study groups was carried out using the Student t-test and Chi-square test. Pearson's correlation coefficients were used to determine the relationships between parameters. The analysis was done by Statistical Package for the Social Sciences (SPSS) 16.0 version software. $\mathrm{P}<0.05$ was considered as statistically significant.

\section{Results}

In this study, we included 36 diagnosed cases of SLE which were referred to the Biochemistry Laboratory from the Dermatology department. Thirty age and sex-matched healthy control were also enrolled in the study. Mean age of SLE patients was $34 \pm 13.49$ years whereas that of control was $36.23 \pm 15.34$ years and was not statistically significant which fulfilled the agematched criteria for selecting the two groups. There were $86 \%$ of females in SLE patients and $80 \%$ female in the control group and the difference was not significant. We did not observe any significant difference in BMI between the groups $(p=0.062)$. CRP level was quite high $(12.8 \pm 12.2 \mathrm{mg} / \mathrm{dL})$ in SLE group as compare to the control $(3 \pm 2.8 \mathrm{mg} / \mathrm{dl})$ and was statistically significant $(p=0.02)$. No significant difference was seen in Hemoglobin and ESR level among the two groups. Serum ADA activity in SLE group (37.03 $\pm 13.03 \mathrm{IU} / \mathrm{L})$ was higher as compared to control $(18.35 \pm 7.71 \mathrm{IU} / \mathrm{L})$ and the difference was statistically significant $(p<0.001)$. The median ANA and anti-dsDNA in the SLE group was $16.9(8.5,42.8)$ and $24.7(20.5,45.0)$, respectively. Serum ANA was positive in 25 out of 36 cases, anti-dsDNA was positive in 17 out of 36 cases whereas ADA was positive in 29 out of 36 cases. A weak positive correlation between ADA and anti-dsDNA ( $r=0.46, p=0.005)$ was observed whereas a moderate correlation between ADA and ANA ( $r=0.525, p=0.001)$ was observed in SLE patients (Fig. 1, 2).

\section{Discussion}

The exact pathophysiology of SLE is not known, although many etiologies like infection, genetic, immunologic etc., have been described [16]. In this study, we investigated the ADA activity in SLE patients and found that its level was significantly high as compared to healthy control. Previous studies have also reported increase serum ADA activity in various diseases, including infection, malignancies and liver disease [17]. Various studies in the past have reported high ADA activity in SLE patients $[10,11,18]$, our results are also in accordance with them. Even though the mechanism by which ADA activity increases has not been fully elucidated, 


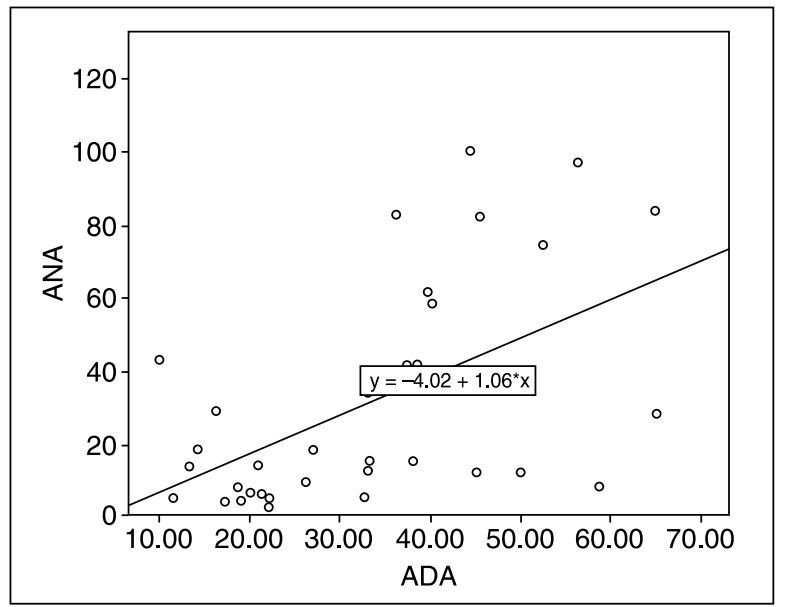

Figure 1. Correlation between adenosine deaminase and antinuclear antibodies in systemic lupus erythematosus patients

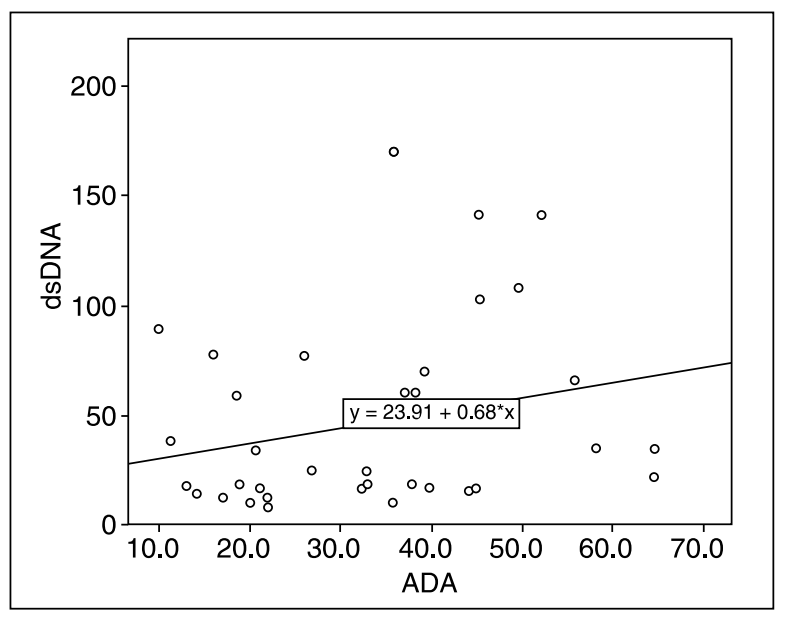

Figure 2. Correlation between adenosine deaminase and anti-double-stranded deoxyribonucleic acid in systemic lupus erythematosus patients possibly its increase in SLE patients can be correlated to altered immune response $[19,20]$.

As per literature review female are more prone to autoimmune diseases as compared to male. A similar finding was observed in our study as well, where $86 \%$ of the SLE patients were female. Laxminarayana et al. [21] have reported that female was having 6-10 fold higher chance of acquiring SLE compared to male.

At present,ANA and anti-dsDNA are an important parameters in the diagnosis of SLE, as per the American Rheumatology Association (ARA) criteria. Though many more criteria are included to complete the diagnosis. In this study serum, ANA and anti-dsDNA in SLE patients was also evaluated. We observed ANA was positive in $69 \%$ of the cases, anti-dsDNA was positive in only $47 \%$ of the cases whereas ADA was positive in $80 \%$ of the cases. This results highlight the utility of ADA in diagnosing SLE as it is more specific than the conventional antibodies.

\section{Conclusion}

This study concludes that serum ADA activity was higher in SLE patients as compared to control group and is more specific in diagnosing the SLE as compared to autoantibodies. Determination of ADA levels is a reliable, cost-effective and easy test that can be used as alternative parameters representing disease activity in SLE patients. However, it would be too early to conclude ADA as a marker of SLE, therefore, further studies with a large number of samples is required to generalize our findings.

\section{Acknowledgements}

The authors acknowledge the technical support from BP Koirala Institute of Health Sciences, Dharan,

Table 1. Basic and laboratory parameter among the groups

\begin{tabular}{llll}
\hline Variables & SLE patients & Control & P value \\
\hline Age (years) & $34 \pm 13.49$ & $36.23 \pm 15.34$ & 0.590 \\
Gender, $\mathrm{n}(\%)$ & & \\
Male & $5(13.8)$ & $6(20)$ & 0.630 \\
Female & $31(86.2)$ & $24(80)$ & \\
$\mathrm{BMI}\left(\mathrm{kg} / \mathrm{m}^{2}\right)$ & $26.2 \pm 4.3$ & $24.4 \pm 3.5$ & 0.063 \\
$\mathrm{Hb}(\mathrm{g} / \mathrm{dL})$ & $11.3 \pm 1.4$ & $10.9 \pm 0.6$ & 0.06 \\
ESR $(\mathrm{mm} / \mathrm{hr})$ & $33.7 \pm 23.5$ & $28.1 \pm 10.3$ & 0.08 \\
CRP $(\mathrm{mg} / \mathrm{L})$ & $12.8 \pm 12.2$ & $3 \pm 2.8$ & 0.02 \\
ADA $(\mathrm{IU} / \mathrm{L})$ & $37.03 \pm 13.03$ & $18.35 \pm 7.71$ & $<0.001$ \\
ANA (U/mL) & $16.9(8.5,42.8)$ & - & $\mathrm{NA}$ \\
dsDNA (IU/L) & $24.7(20.5,45.0)$ & - & $\mathrm{NA}$
\end{tabular}

ADA — adenosine deaminase; ANA — antinuclear antibodies; BMI — body mass index; dsDNS — double-stranded deoxyribonucleic acid; SLE - systemic lupus erythematosus 
Nepal. Authors are also grateful to the lab staffs for helping in the analysis of the sample and to the study participants who gave their consent to participate in the study.

\section{Conflict of interest}

The authors report no competing interests.

\section{References}

1. Franco R, Valenzuela A, Lluis $\mathrm{C}$, et al. Enzymatic and extraenzymatic role of ecto-adenosine deaminase in lymphocytes. Immunol Rev. 1998; 161: 27-42, doi: 10.1111/j.1600-065x.1998.tb01569.x, indexed in Pubmed: 9553762.

2. Whitmore KV, Gaspar HB. Adenosine deaminase deficiency - more than just an immunodeficiency. Front Immunol. 2016; 7: 314, doi: 10.3389/fimmu.2016.00314, indexed in Pubmed: 27579027.

3. Kwan A, Abraham RS, Currier R, et al. Newborn screening for severe combined immunodeficiency in 11 screening programs in the United States. JAMA. 2014; 312(7): 729-738, doi: 10.1001/jama.2014.9132, indexed in Pubmed: 25138334.

4. Cicalese MP, Ferrua F, Castagnaro L, et al. Update on the safety and efficacy of retroviral gene therapy for immunodeficiency due to adenosine deaminase deficiency. Blood. 2016; 128(1): 45-54, doi 10.1182/blood-2016-01-688226, indexed in Pubmed: 27129325.

5. Ferrua F, Brigida I, Aiuti A. Update on gene therapy for adenosine deaminase-deficient severe combined immunodeficiency. Curr Opin Allergy Clin Immunol. 2010; 10(6): 551-556, doi: 10.1097/ACl.0b013e32833fea85, indexed in Pubmed: 20966749.

6. Sauer AV, Morbach H, Brigida I, et al. Defective B cell tolerance in adenosine deaminase deficiency is corrected by gene therapy. J Clin Invest. 2012; 122(6): 2141-2152, doi: 10.1172/JCl61788, indexed in Pubmed: 22622038.

7. Sauer AV, Brigida I, Carriglio N, et al. Alterations in the adenosine metabolism and CD39/CD73 adenosinergic machinery cause loss of Treg cell function and autoimmunity in ADA-deficient SCID. Blood. 2012; 119(6): 1428-1439, doi: 10.1182/blood-2011-07-366781, indexed in Pubmed: 22184407.

8. Nalesnik M, Nikolić JM, Jandrić S. Adenosine deaminase and C-reactive protein in diagnosing and monitoring of rheumatoid arthritis. Med Glas (Zenica). 2011; 8(1): 163-168, indexed in Pubmed: 21263416.
9. Vinapamula KS, Pemmaraju SV, Bhattaram SK, et al. Serum Adenosine Deaminase as Inflammatory Marker in Rheumatoid Arthritis. J Clin Diagn Res. 2015; 9(9): BC08-BC10, doi: 10.7860/JCDR/2015/14296.6483, indexed in Pubmed: 26500897.

10. Hitoglou S, Hatzistilianou M, Gougoustamou D, et al. Adenosine deaminase activity and its isoenzyme pattern in patients with juvenile rheumatoid arthritis and systemic lupus erythematosus. Clin Rheumatol. 2001; 20(6): 411-416, doi: 10.1007/s100670170005, indexed in Pubmed: 11771524

11. Saghiri R, Ghashghai N, Movaseghi S, et al. Serum adenosine deaminase activity in patients with systemic lupus erythematosus: a study based on ADA1 and ADA2 isoenzymes pattern. Rheumato Int. 2012; 32(6): 1633-1638, doi: 10.1007/s00296-011-1836-8, indexed in Pubmed: 21350874

12. Vasudha KC, Kumar AN, Venkatesh T. Studies on the age dependent changes in serum adenosine deaminase activity and its changes in hepatitis. Indian J Clin Biochem. 2006; 21(1): 116-120, doi: 10.1007/BF02913078, indexed in Pubmed: 23105581.

13. Samsonov MY, Tilz GP Egorova $O$, et al. Serum soluble markers of immune activation and disease activity in systemic lupus erythematosus. Lupus. 1995; 4(1): 29-32, doi: 10.1177/096120339500400107. indexed in Pubmed: 7767335.

14. Laxminarayana D, O'Rourke KS, Maas S, et al. Altered editing in RNA editing adenosine deaminase ADAR2 gene transcripts of systemic lupus erythematosus T lymphocytes. Immunology. 2007; 121(3): 359-369, doi: 10.1111/j.1365-2567.2007.02582 x indexed in Pubmed: 17376196.

15. Petri M. Hopkins lupus cohort. 1999 update. Rheum Dis Clin North Am. 2000; 26(2): 199-213, v, doi: 10.1016/s0889-857x(05)70135-6, indexed in Pubmed: 10768209.

16. Currey HLF. Aetiology and pathogenesis of rheumatoid arthritis. In: Copeman's Textbook of the rheumatic diseases. Churchill Livingstone, Edinburgh 1978: 261-272.

17. Ungerer JP, Oosthuizen HM, Bissbort $\mathrm{SH}$, et al. Serum adenosine deaminase: isoenzymes and diagnostic application. Clin Chem. 1992; 38(7): 1322-1326, indexed in Pubmed: 1623598.

18. Taysi S, Polat MF Sari RA et al Serum adenosine deaminase and cytidine deaminase activities in patients with systemic lupus erythematosus. Clin Chem Lab Med. 2002; 40(5): 493-495, doi: 10.1515/CCLM.2002.085, indexed in Pubmed: 12113294.

19. Fischer $D$, Van der Weyden MB, Snyderman $R$, et al. A role for adenosine deaminase in human monocyte maturation. J Clin Invest. 1976 58(2): 399-407, doi: 10.1172/JCl108484, indexed in Pubmed: 956374

20. Gakis $C$. Adenosine deaminase (ADA) isoenzymes ADA1 and ADA2: diagnostic and biological role. Eur Respir J. 1996; 9(4): 632-633, doi: 10.1183/09031936.96.09040632, indexed in Pubmed: 8726922.

21. Laxminarayana D, O'Rourke KS, Maas S, et al. Altered editing in RNA editing adenosine deaminase ADAR2 gene transcripts of systemic lupus erythematosus T lymphocytes. Immunology. 2007; 121(3) 359-369, doi: 10.1111/j.1365-2567.2007.02582.x, indexed in Pubmed: 17376196 